\title{
Fibulin-3 as a Diagnostic Biomarker in Patients with Malignant Mesothelioma
}

\author{
Halide Kaya ${ }^{1 *}$, Melike Demir ${ }^{1}$, Mahsuk Taylan ${ }^{1}$, Cengizhan Sezgi ${ }^{1}$, Abdullah \\ Cetin Tanrikulu', Sureyya Yilmaz', Mehmet Bayram², Ibrahim Kaplan ${ }^{3}$, \\ Abdurrahman Senyigit ${ }^{1}$
}

\begin{abstract}
Background: New tumour biomarkers are being intensely investigated for malignant mesothelioma (MM). Fibulin-3 is produced in MM but its role remains uncertain. The aim of this study was to evaluate the validity of measuring serum fibulin-3 in the diagnosis and prognosis of MM. Materials and Methods: This prospective study was performed on 43 patients and 40 healthy controls who were admitted to our hospital between January 2012 and January 2014. Data from MM patients, including demographic and clinical features, routine laboratory data, levels of serum fibulin-3, and treatment outcomes were defined as potential prognostic factors. The receiver operating characteristic (ROC) curve for fibulin-3 was used to detect the cut-off value with highest sensitivity and specificity. Univariate survival analysis was performed using the Kaplan-Meier method in patients with MM. Afterwards, the possible factors identified with univariate analyses were entered into the cox regression analysis. Results: Our results revealed that patients with MM had significantly higher serum levels of fibulin-3 than controls. The results showed that the best cut-off point was $36.6 \mathrm{ng} / \mathrm{ml}$ with an AUC (area under the curve) $=0.976$, sensitivity $=93.0 \%$ and specificity $=90.0$. In our study, the initial significant poor prognostic factors were advanced stage, high white blood cell count, high platelet count, high $\mathrm{C}$-reactive protein ( $\mathbf{p}<0.05$ for each variable). Later, according to multivariate analysis the results showed only advanced stage as significant parameter $(p=0.040)$. Conclusions: We determined that real use for serum fibulin-3 was not for prognosis but for diagnosis in MM. Also advanced stage was associated with poor MM prognosis.
\end{abstract}

Keywords: Malignant mesothelioma - serum fibulin-3 - diagnosis - prognosis

Asian Pac J Cancer Prev, 16 (4), 1403-1407

\section{Introduction}

Malignant mesothelioma (MM) is a lethal tumor that typically has a poor prognosis. The greatest risk factor for the development of MM is asbestos exposure, either occupational or environmental. Asbestos exposure is identified in $50-90 \%$ of the patients with MM. Contrary to developed countries, the vast majority of mesothelioma in developing countries is secondary to environmental asbestos exposure (Senyigit et al., 2000; Metintas et al., 2002; Berk et al., 2013).

Early detection is limited because there is a long delay between the onset of symptoms and the diagnosis of MM, an inability of imaging to detect the disease at an early stage, and lack of sensitive and specific blood-based markers. The difficulties in establishing the diagnosis of mesothelioma were explained in a large scale study (Boutin et al., 1993). Therefore, most patients present in an advanced stage of this disease because it is an insidious and relatively nonspecific clinical presentation.
Histopathological examination is required for the definitive diagnosis of the MM. The differential diagnosis of MM includes both benign and malignant processes, especially the differential diagnosis between mesothelioma and adenocarcinoma is difficult. Therefore establishing a tissue diagnosis can be difficult (Ordonez 2003). Mesothelioma patients who are treated with supportive care have a median survival of 4 to 13 months (Anthman 1993). Even those treated with the best available chemotherapy survive 13-25 months (Vogelzang et al., 2003).

Recently, research has focused on evaluating several less invasive biomarkers for MM screening, diagnosis, prognostication and monitoring. It has been well documented that soluble mesothelin-related protein, the most extensively studied blood-based mesothelioma biomarker, is limited by a high specificity but poor sensitivity for early diagnosis of MM (Creaney and Robinson, 2009; Hollevoet et al., 2012). Unfortunately, an ideal biomarker for MM has not been yet identified. 
A new biomarker, fibulin-3, has recently been reported as a potential biomarker to facilitate the identification of patients with mesothelioma. Fibulin-3 is an extracellular glycoprotein that is encoded by the epidermal growth factor-containing fibulin-like extracellular matrix protein 1 (EFEMP1) gene and its expression is low in normal tissues. Fibulin-3 is expressed in condensing mesenchyme, giving rise to bony and cartilaginous structures (Zhang and Marmorstein, 2010). In previous studies, different types of cancer have been associated with fibulin-3, such as brain, hepatocellular, prostate, colorectal, and lung (Hu et al., 2009; Nomoto et al., 2010; Kim et al., 2011; Tong et al., 2011; Kim et al, 2012) however, there are few studies pertaining to the association with MM (Pass et al., 2012; Agha et al., 2014; Creaney et al., 2014). In these studies, levels of fibulin-3 investigated in patients with occupational asbestos exposure. But yet, the precise role of fibulin-3 in MM is still under investigation.

The aim of this prospective study was to determine the diagnostic and prognostic value of fibulin-3 and as well as known clinical prognostic factors in MM. This is the first study, to our knowledge, focusing on patients with a history of environmental asbestos exposure.

\section{Materials and Methods}

This was a prospective study on 43 patients with biopsy-proven MM who were admitted to our hospital between January 2012 and January 2014. Additionally, 40 healthy controls were included; they had normal chest $\mathrm{X}$-rays and no history of environmental asbestos exposure. Informed consent was obtained from each participant. The study was approved by the local Ethics Committee. Data from MM patients, including demographic and clinical features routine laboratory investigations, levels of fibulin-3, and treatment outcomes were defined as potential prognostic factors and were recorded at the time of diagnosis.

\section{Clinical Features of Mesothelioma Patients}

The following pre-treatment patient characteristics were analyzed for their effect on survival age at the time of diagnosis ( $\leq 60 />60$ years), gender, body mass index (BMI) according to weight and height information, environmental asbestos exposure (yes or no), duration of exposure ( $\leq 20 />20$ years), smoking history and pack-years of smoking ( $\leq 20 />20)$, symptoms at the time of diagnosis [presence of dyspnea, chest pain, abdominal pain, weight loss ( $>5 \%$ in the last 3 months)], the interval between the onset of symptoms until the diagnosis ( $\leq 6$ or $>6$ months), Karnofsky Performance Status ( $\leq 60 />60 \%)$, primary site of pleural tumor (right/left), presence of pleural fluid and cytology, diagnostic procedures [operative (video-assisted thoracoscopic surgery, minithoracotomy or non-operative (percutaneous pleural/peritoneal needle biopsy)], histopathological types [epithelioid or others (biphasic sarcomatous and unspecified)], staging system according to International Union Against Cancer Classification [stage I-II (earlier) or stage III-IV (advanced)], treatment approach (three groups: best supportive care, systemic chemotherapy, or multimodal therapy), follow-up time (the interval between the diagnosis and death or the last visit) and patient status (alive or dead).

\section{Laboratory results}

The upper limit of the laboratory reference range of different tests is as follows: hemoglobin concentration $(\mathrm{Hb}$, $<12.3$ or $\geq 12.3 \mathrm{~g} / \mathrm{dl}$ ), white blood cell count (WBC, $\leq 8.3$ or $>8.3 / \mu \mathrm{l})$ platelet count $(\leq 400.0$ or $>400.0 / \mu \mathrm{l})$, levels of serum albumin $(\leq 3.0$ or $>3.0 \mathrm{~g} / \mathrm{dl})$, lactate dehydrogenase (LDH, $\leq 500$ or $>500 \mathrm{U} / \mathrm{l}$ ), C-reactive protein (CRP, $\leq 50$ or $>50 \mathrm{mg} / \mathrm{l}$ ), and erythrocyte sedimentation rate (ESR, $\leq 50$ or $>50 \mathrm{~mm} / \mathrm{h}$ ).

\section{Collection of blood samples for fibulin-3 measurement}

Ten milliliters of venous blood samples were obtained from all participants. They were allowed to clot for 30 minutes at room temperature before they were centrifuged at $4000 \mathrm{rpm}$ and $+4^{\circ} \mathrm{C}$ for 10 minutes. Following this, the obtained sera were transferred into microcentrifuge tubes and later serum samples were stored $-80^{\circ} \mathrm{C}$ until analyzed. Fibulin-3 levels of patient and control groups were determined using commercially available the enzymelinked immunosorbent assay (ELISA) kit according to the manufacturers' protocols.

\section{Statistical analysis}

The data collected were tabulated and analyzed by using the SPSS ${ }^{\circledR}$ for Windows version 15.0. Characteristics were reported using frequency and descriptive analysis. The Student t-test (parametric) or Mann-Whitney U test (nonparametric) was used to compare two independent subgroups, as appropriate. The receiver operating characteristic (ROC) curve for fibulin-3 was used to detect the cutoff value with highest sensitivity and specificity Sensitivity, specificity, positive and negative predictive value (PPV and NPV, respectively) and diagnostic accuracy were calculated. Survival was defined as the period between the date of the pathological diagnosis and death. For patients who did not die, this duration was defined as the period between the time of diagnosis and January 2014. Univariate survival analysis was performed using the Kaplan-Meier method in patients with MM. Afterwards, the possible factors identified with univariate analyses were entered into the Cox Regression analysis, to determine independent predictors of survival. $P$ values $<0.05$ were considered statistically significant.

\section{Results}

\section{Patient characteristics}

Baseline characteristics for the 43 patients (primary involvement: 39 pleural, 4 peritoneal mesothelioma) are summarized in Table 1.

\section{Diagnostic evaluation}

When MM patients and healthy controls were compared, there was no significant difference among age, gender, history of smoking and BMI. However, serum fibulin-3 levels [mean $( \pm$ SD) $90.3 \pm 42.1$ and $17.8 \pm 12.7$ ng per mililiters, respectively] differed significantly $(\mathrm{p}<0.001)$. The ROC curve was generated to analyze the 
diagnostic values of serum fibulin-3 (Figure 1). The results showed (Table 2) that the best cut-off point for serum fibulin-3 was $36.6 \mathrm{ng} / \mathrm{ml}$ with an AUC (area under the curve $)=0.976$, sensitivity $=93.0 \%$, and specificity $=90.0$.

\section{Survival analyses}

Parameters of the clinical and laboratory that we expected to be associated with prognosis were used in univariate analysis. We found that significant poor prognostic factors were advanced stage, high WBC, high platelet count, high CRP ( $\mathrm{p}<0.05$ for each variable). No associations were found between MM prognosis and age, gender, asbestos exposure and duration of exposure,

Table 1. Characteristics of the Malignant Mesothelioma Patients

\begin{tabular}{|c|c|}
\hline Age (mean \pm sd, years) & $61.7 \pm 12.2$ \\
\hline Age group $(\leq 60 />60$ years $)$ & $19 / 24$ \\
\hline Gender (female/male) & $22 / 21$ \\
\hline $\mathrm{BMI}\left(\mathrm{mean} \pm \mathrm{sd}, \mathrm{kg} / \mathrm{m}^{2}\right)$ & $24.8 \pm 4.2$ \\
\hline Asbestos exposure $(\mathrm{n}, \%)$ & $36(83.7)$ \\
\hline Duration of asbestos exposure (mean \pm sd, years) & $27.7 \pm 18.7$ \\
\hline History of smoking $(\mathrm{n}, \%)$ & $20(46.5)$ \\
\hline Duration of symptoms (months) & $5.9 \pm 6.4$ \\
\hline \multicolumn{2}{|l|}{ Laboratory results (mean \pm sd) } \\
\hline Hemoglobin concentration $(\mathrm{g} / \mathrm{dl})$ & $12.5 \pm 1.9$ \\
\hline White blood cell count $\left(\times 10^{3} / \mu 1\right)$ & $9.2 \pm 3.7$ \\
\hline Platelet count $\left(\times 10^{3} / \mu 1\right)$ & $346.8 \pm 174.6$ \\
\hline Serum albumin level (g/dl) & $3.2 \pm 0.6$ \\
\hline Lactate dehydrogenase (U/l) & $238.0 \pm 95.3$ \\
\hline C-reactive protein $(\mathrm{mg} / \mathrm{l})$ & $45.1 \pm 50.2$ \\
\hline Erythrocyte sedimentation rate $(\mathrm{mm} / \mathrm{h})$ & $33.4 \pm 16.8$ \\
\hline Levels of fibulin-3 (ng/ml) & $90.3 \pm 42.1$ \\
\hline Diagnostic method (n, operative/nonoperative) & $23 / 20$ \\
\hline \multicolumn{2}{|l|}{ Histopathological type $(\mathrm{n}, \%)$} \\
\hline Epithelial & $30(69.8)$ \\
\hline Others & $13(30.2)$ \\
\hline \multicolumn{2}{|l|}{ Stage $(n, \%)(n=39)$} \\
\hline Stage I-II & $18(46.1)$ \\
\hline Stage III-IV & $21(53.9)$ \\
\hline Performance status (Karnofsky) $(\mathrm{n}, \leq 60 />60 \%)$ & $9 / 33$ \\
\hline \multicolumn{2}{|l|}{ Treatment $(\mathrm{n}, \%)$} \\
\hline Best supportive care & 21(49) \\
\hline Chemotherapy & $15(35)$ \\
\hline Multimodal therapy & $7(16)$ \\
\hline Survival time (mean \pm sd, months) & $9.6 \pm 9.5$ \\
\hline \multicolumn{2}{|l|}{ Status $(\mathrm{n}, \%)$} \\
\hline Alive & $20(46.5)$ \\
\hline Dead & $23(53.5)$ \\
\hline
\end{tabular}

*BMI, Body mass index

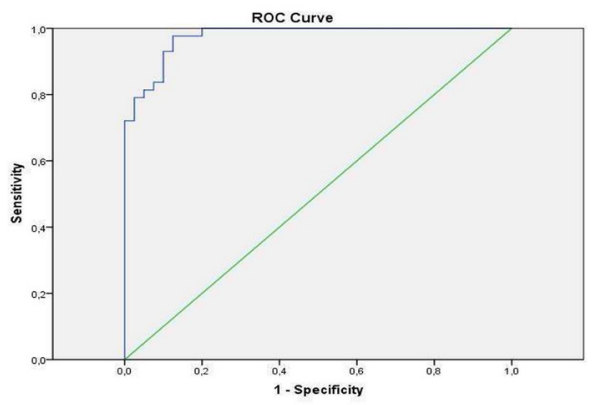

Figure 1. ROC Curve to Analyze the Diagnostic Values of Serum Fibulin-3
Table 2. Validity of Different Serum Fibulin-3 Levels for the Diagnosis of the Mesothelioma

\begin{tabular}{lccccc}
\hline $\begin{array}{l}\text { Cut-off } \\
\text { point }\end{array}$ & $\begin{array}{c}\text { Sensitivity } \\
(\%)\end{array}$ & $\begin{array}{c}\text { Specificity } \\
(\%)\end{array}$ & $\begin{array}{c}\text { PPV } \\
(\%)\end{array}$ & $\begin{array}{c}\text { NPV } \\
(\%)\end{array}$ & $\begin{array}{c}\text { Accuracy } \\
(\%)\end{array}$ \\
\hline 30.1 & 97.7 & 87.5 & 97.2 & 89.4 & 92.8 \\
36.6 & 93.0 & 90.0 & 92.3 & 90.9 & 91.6 \\
48.8 & 81.4 & 95.0 & 82.6 & 94.6 & 87.9 \\
\hline
\end{tabular}

*PPV, Positive predictive value; NPV, Negative predictive value

Table 3. Kaplan-meier Survival Analysis of 43 Mesothelioma Patients

\begin{tabular}{llrl}
\hline Variables & \multicolumn{3}{c}{$\begin{array}{c}\text { Median survival } \\
\text { (months) }\end{array}$} \\
& \multicolumn{3}{c}{$\mathrm{p}^{*}$} \\
\hline Age group (years) & $\leq 60$ & 17.0 & $\mathrm{NS}$ \\
& $>60$ & 7.0 & \\
Gender & Female & 12 & $\mathrm{NS}$ \\
& Male & 12 & \\
Stage & I-II & 21 & 0.004 \\
& III-IV & 7 & \\
White blood cell count $\left(\mathrm{x} 10^{3} / \mu 1\right)$ & $\leq 8.3$ & 21 & 0.035 \\
& $>8.3$ & 9 & \\
Platelet count $\left(\mathrm{x} 10^{3} / \mu \mathrm{l}\right)$ & $\leq 400.0$ & 17 & 0.004 \\
& $>400.0$ & 7 & \\
C-reactive protein $(\mathrm{mg} / \mathrm{l})$ & $\leq 50$ & 17 & 0.029 \\
& $>50$ & 9 & \\
\hline
\end{tabular}

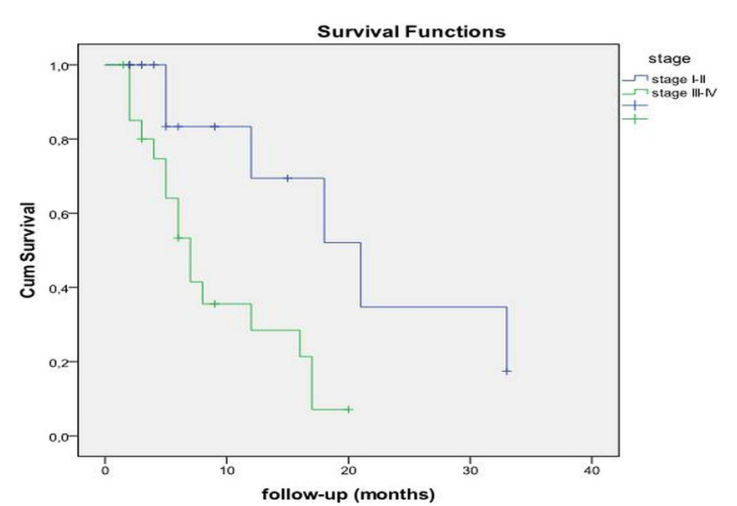

Figure 2. Multivariate Analysis Results for Advanced Stage Showing Increased Poor Prognosis

smoking history, initial symptoms, histopathologic subtype, performance status and serum levels of $\mathrm{Hb}$, albumin, LDH, ESR and serum fibulin-3 (p>0.05 for each variable). Variables with $\mathrm{p}<0.05$ in univariate analysis were used in the final model for multivariate analysis.

\section{Prognostic factors affecting survival}

Results of the univariate analyses revealed (Table 3) that advanced stage, high WBC, high platelet count, and high CRP were associated with poor MM prognosis. However, then the possible factors identified with univariate analyses were entered subsequently into the cox regression analysis, and only advanced stage remained as an independent predictor of survival $(\mathrm{p}=0.040)$. According to multivariate analysis results, advanced stage (Figure 2) increased poor prognosis 3.9 times.

\section{Discussion}

In this study, we found that levels of fibulin-3 in serum may aid in determining the diagnosis of mesothelioma. 
However, it is not associated with their prognosis. Also, when the relationship between survival and parameters of the clinical and laboratory was investigated poor MM prognosis was associated with advanced stage.

It is well known that people have been exposed to environmental asbestos in some rural areas of the Southeast Anatolia Region of Turkey due to its geographical structure. So environmental asbestos exposure starts at birth and continues (Senyigit et al., 2000) Occupational asbestos exposures were more frequent in men, whereas environmental asbestos exposure was found to be nearly as frequent in men and women (Metintas et al., 2002). While pleural mesothelioma after occupational asbestos exposure mainly effects men (male to female ratio: 5 to 1 ), male/female ratio is much smaller in non-occupational mesothelioma patients (male to female ratio: 1 to 1 ) (Metintas et al., 2008; Teta et al., 2008). In our study results shown in Table 1 , the ratio of male-to-female mesothelioma patients was approximately equal. Most of our patients declared to previously reside in rural areas and had environmental asbestos exposure for many years. Furthermore, there was no difference between sexes in the duration of exposure.

Historically, biomarkers have not turned out to be all that useful in predicting mesothelioma diseases (Creaney and Robinson, 2000). Gold standard for definitive diagnosis is still histopathological diagnosis, however, sometimes can be very difficult to achieve diagnosis (Ordonez, 2003). In other words, especially in such cases without histopathologic diagnosis, biomarkers provide additional contribution. Promising new biomarker studies are ongoing. In regards to diagnosis of MM, few studies have researched fibulin-3 in serum or plasma and in pleural effusions in order to distinguish mesothelioma patients from either healthy controls or patients with benign disease (Pass et al., 2012; Agha et al., 2014).

Pass et al. (2012) were the first to report that that they measured plasma fibulin-3 levels (from 92 patients with mesothelioma, 136 asbestos-exposed persons without cancer, 93 patients with effusions not due to mesothelioma, and 43 healthy controls), effusions (from 74 patients with mesothelioma, 39 with benign effusions, and 54 with malignant effusions not due to mesothelioma), or both (Pass et al., 2012). They found that plasma fibulin-3 levels did not vary according to age, sex, duration of asbestos exposure, or degree of radiographic changes and that plasma fibulin-3 levels were significantly higher in patients with pleural mesothelioma $(105 \pm 7 \mathrm{ng} /$ $\mathrm{ml}$ in the Detroit cohort and $113 \pm 8 \mathrm{ng} / \mathrm{ml}$ in the New York cohort) than in asbestos-exposed persons without mesothelioma ( $14 \pm 1 \mathrm{ng} / \mathrm{ml}$ and $24 \pm 1 \mathrm{ng} / \mathrm{ml}$ respectively). They found that elevated levels of fibulin- 3 in the plasma had a high sensitivity and specificity ( 97 and 96 percent, respectively, at a cutoff value of $52.8 \mathrm{ng} / \mathrm{ml}$ of fibulin-3) in distinguishing patients with pleural mesothelioma from those with a history of asbestos exposure but without mesothelioma and from those with other malignancies or benign causes of pleural effusion (Pass et al., 2012). The findings of this study suggest that levels of fibulin-3 in plasma and effusions may aid in determining the diagnosis and prognosis of pleural mesothelioma.
Another study has confirmed the role of serum and pleural fibulin-3 in the diagnosis of pleural mesothelioma and its role in differentiating between malignant and benign pleural diseases (Agha et al., 2014). This study showed correlation between serum and pleural fibulin-3. It showed that using a cut-off point of serum fibulin-3 (18 $\mathrm{ng} / \mathrm{ml}$ ), with AUC of 0.931 (sensitivity $100 \%$, specificity $77.8 \%$ ) differentiation between MM and benign pleural effusion could occur.

Our results showed that the levels of serum fibulin-3 in mesothelioma patients were significantly higher than healthy subjects $(90.3 \pm 42.1$ and $17.8 \pm 12.7 \mathrm{ng}$ per mililiters respectively). Also, the best cut-off point of serum fibulin-3 (36.6 ng/ml) had the highest sensitivity (sensitivity $93 \%$, specificity $90 \%$ ). In patients presenting with a suspected mesothelioma, high levels of fibulin-3 $(>36.6 \mathrm{ng} / \mathrm{ml})$ in serum indicate which patients need further invasive procedures. In our study, we found a diagnostic value of serum fibulin-3, but it has not contributed to estimating mesothelioma prognosis. Although our study levels of fibulin-3 in mesothelioma patients were not associated with the prognosis, this result may be due to the small sample size.

Prognostic scoring systems may provide useful information in MM. The two most widely used scoring systems were developed by the Cancer and Leukemia Group B (CALGB) and the European Organization for Research and Treatment of Cancer (EORTC). When jointly evaluated, these studies show that pleural (as compared to peritoneal or pericardial) involvement, age older than 75 years, male gender, poor performance status, chest pain, non epithelial histology, advanced stage, leucocytosis, thrombocytosis and elevated LDH have predicted poor survival (Edwards et al., 2000; Metintas et al., 2001).

Recently, elevated serum CRP levels in several malignant diseases including breast, ovarian, renal, and lung cancer have also been identified as negative prognostic indicators (Hefler et al., 2008; Pierce et al., 2009; Wang and Sun, 2009; O'Dowd et al., 2010). With respect to $\mathrm{MM}$, two retrospective screening studies suggested CRP as potent prognostic marker. High levels of serum CRP have been associated with poor prognosis due to the role of chronic inflammation in the development of MM that has been well established (Tanrikulu et al., 2010; Nojiri et al., 2011). A major limitation of all of the staging systems is the difficulty in assessing the extent of disease prior to treatment. Early stage was considered among the important positive prognostic characteristics in some studies (Van Gelder et al., 1994; Rusch and Venkatraman, 1999). In other study, advanced clinical stage was related to survival (Komurcuoglu et al., 2014). In our study, the initial significant poor prognostic factors were advanced stage, high WBC, high platelet count, high CRP ( $\mathrm{p}<0.05$ for each variable). Later according to multivariate analysis the results showed only advanced stage as significant parameter.

The small sample size limited the study. Additionally, cancer-free patients with asbestos exposure and adenocarcinoma patients were not placed in separate groups. 
In conclusion, we determined that real use for serum fibulin-3 was not for prognosis but for diagnosis in MM. Also advanced stage was associated with poor MM prognosis. Further studies comparing fibulin-3 with other common biomarkers for MM should be done in order to confirm the role of fibulin-3 in the diagnosis, treatment monitoring and evaluation of disease prognosis. Consequently, prospective studies with a larger sample size are needed to rigorously assess the diagnostic power of fibulin-3.

\section{Acknowledgements}

This submission has not been published anywhere previously and is not simultaneously being considered for any other publication. The authors report no conflict of interests.

\section{References}

Agha MA, El-Habashy MM, El-Shazly RA (2014). Role of fibulin-3 in the diagnosis of malignant mesothelioma. Egyptian J Chest Diseases Tuberculosis, 63, 99-105.

Antman KH (1993). Natural history and epidemiology of malignant mesothelioma. Chest, 103, 373-6.

Berk S, Dogan OT, Kilickap S, et al (2013). Clinical characteristics, treatment and survival outcomes in malignant mesothelioma: Eighteen years' experience in Turkey. Asian Pac J Cancer Prev, 13, 5735-9.

Boutin C, Rey F, Gouvernet J, et al (1993). Thoracoscopy in pleural malignant mesothelioma: a prospective study of 188 consecutive patients. Part 2: Prognosis and staging. Cancer, 72, 394-404.

Creaney J, Robinson BW (2009). Serum and pleural fluid biomarkers for mesothelioma. Curr Opin Pulm Med, 15, 366-70.

Creaney J, Dick IM, Meniawy TM, et al (2014). Comparison of fibulin-3 and mesothelin as markers in malignant mesothelioma. Thorax, 69, 895-902.

Edwards JG, Abrams KR, Leverment JN, et al (2000). Prognostic factors for malignant mesothelioma in 142 patients: validation of CALGB and EORTC prognostic scoring systems. Thorax, 55, 731-5.

Hefler LA, Concin N, Hofstetter G, et al (2008). Serum $\mathrm{C}$-reactive protein as independent prognostic variable in patients with ovarian cancer. Clin Cancer Res, 14, 710-4.

Hollevoet K, Reitsma JB, Creaney J, et al (2012). Serum mesothelin for diagnosing malignant pleural mesothelioma: an individual patient data meta-analysis. J Clin Oncol, 30, 1541-9.

Hu B, Thirtamara-Rajamani KK, Sim H, Viapiano MS (2009). Fibulin-3 is uniquely upregulated in malignant gliomas and promotes tumor cell motility and invasion. Mol Cancer Res, 7, 1756-70

Kim EJ, Lee SY, Woo MK, et al (2012). Fibulin-3 promoter methylation alters the invasive behavior of non-small cell lung cancer cell lines via MMP-7 and MMP-2 regulation. Int J Oncol, 40, 402-8.

Kim YJ, Yoon HY, Kim SK, et al (2011). EFEMP1 as a novel DNA methylation marker for prostate cancer: array-based DNA methylation and expression profiling. Clin Cancer Res, 17, 4523-30.

Komurcuoglu B, Cirak AK, Kirakli SC, et al (2014). Prognostic factors affecting survival in malignant pleural mesothelioma: analysis of 125 subjects. Tumori, 100,55-9.
Metintas M, Metintas S, Ucgun I, et al (2001). Prognostic factors in diffuse malignant pleural mesothelioma: pretreatment clinical and laboratory characteristics. Respir Med, 95, 829-35.

Metintas M, Metintas S, Ak G, et al (2008). Epidemiology of pleural mesothelioma in a population with non-occupational asbestos exposure. Respirology, 13, 117-21.

Metintas S, Metintas M, Ucgun I, Oner U (2002). Malignant mesothelioma due to environmental exposure to asbestos: follow-up of a Turkish cohort living in a rural area. Chest, 122, 2224-9.

Nojiri S, Gemba K, Aoe K, et al (2011). Survival and Prognostic Factors in Malignant Pleural Mesothelioma: A Retrospective Study of 314 patients in the West Part of Japan. Jpn J Clin Oncol, 41, 32-9.

Nomoto S, Kanda M, Okamura Y, et al (2010). Epidermal growth factor-containing fibulin-like extracellular matrix protein 1, EFEMP1, a novel tumor-suppressor gene detected in hepatocellular carcinoma using double combination array analysis. Ann Surg Oncol, 17, 923-32.

O’Dowd C, McRae LA, McMillan DC, Kirk A, Milroy R (2010). Elevated preoperative C- reactive protein predicts poor cancer specific survival in patients undergoing resection for non-small cell lung cancer. J Thorac Oncol, 5, 988-92.

Ordonez NG (2003). The immunohistochemical diagnosis of mesothelioma: a comparative study of epithelioid mesothelioma and lung adenocarcinoma. Am J Surg Pathol, 27, 1031-51.

Pass HI, Levin SM, Harbut MR, et al (2012). Fibulin-3 as a blood and effusion biomarker for pleural mesothelioma. $N$ Engl J Med, 367, 1417-27.

Pierce BL, Ballard-Barbash R, Bernstein L, et al (2009). Elevated biomarkers of inflammation are associated with reduced survival among breast cancer patients. J Clin Oncol, 27, 3437-44.

Rusch VW, Venkatraman ES (1999). Important prognostic factors in patients with malignant pleural mesothelioma, managed surgically. Ann Thorac Surg, 68, 1799-804.

Senyigit A, Babayigit C, Gokirmak M, et al (2000). Incidence of malignant pleural mesothelioma due to environmental asbestos fiber exposure in the southeast of Turkey. Respiration, 67, 610-4.

Tanrikulu AC, Abakay A, Kaplan MA, et al (2010). A clinical, radiographic and laboratory evaluation of prognostic factors in 363 patients with malignant pleural mesothelioma. Respiration, 80, 480-7.

Teta MJ, Mink PJ, Lau E, Sceurman BK, Foster ED (2008). US mesothelioma patterns 1973-2002: indicators of change and insights into background rates. Eur J Cancer Prev, 17, 525-34.

Tong JD, Jiao NL, Wang YX, Zhang YW, Han F (2011). Downregulation of fibulin-3 gene by promoter methylation in colorectal cancer predicts adverse prognosis. Neoplasma, 58, 441-8.

Van Gelder T, Damhuis RAM, Hoogsteden HC (1994). Prognostic factors and survival in malignant pleural mesothelioma. Eur Respir J, 7, 1035-38.

Vogelzang NJ, Rusthoven JJ, Symanowski J, et al (2003). Phase III study of pemetrexed in combination with cisplatin versus cisplatin alone in patients with malignant pleural mesothelioma. J Clin Oncol, 21, 2636-44.

Wang CS, Sun CF (2009). C-reactive protein and malignancy: clinico-pathological association and therapeutic implication. Chang Gung Med J, 32, 471-82.

Zhang Y, Marmorstein LY (2010). Focus on molecules: fibulin-3 (EFEMP1). Exp Eye Res, 90, 374-5. 